\title{
The Use of Doppler Ultrasound to Diagnose Deep Vein Thrombosis of the Lower Limbs
}

\section{Nagla Hussein Mohamed Khalid*}

Department of Diagnostic Radiology, Faculty of Applied Medical Sciences, Najran University, Najran, KSA

*Corresponding Author: Nagla Hussein Mohamed Khalid, Department of Diagnostic Radiology, Faculty of Applied Medical Sciences, Najran University, Najran, KSA.
Received: October 19, 2020

Published: October 28, 2020

(C) All rights are reserved by Nagla Hussein

Mohamed Khalid.

\begin{abstract}
In most cases of pulmonary embolism, death occurs as a result of deep vein thrombosis (DVT) of the lower extremities. Therefore, to avoid DVT complications and sequel, DVT must be diagnosed as early as possible and this can be achieved via B-mode and colour Doppler imaging. The present paper seeks to investigate the suitability of the imaging method of ultrasound for lower extremity DVT diagnosis and to explore the outcomes of the use of this method in DVT cases. To this end, the paper undertakes a retrospective descriptive study of 50 cases of ultrasound-based diagnosis of DVT at King Khalid Hospital in the period between January 2019 and August 2020. Half of the cases were subjected to compression, colour and duplex ultrasound, 14 cases were subjected to compression and duplex ultrasound and 11 cases were subjected to compression and colour Doppler. The mean age across all cases was 46.2 \pm 19.9 years. The majority of cases $(56 \%)$ were in the age range 22 - 41 years old ( $n=28$ ), while $28 \%$ of cases were in the age range 42 - 61 years old $(n=14), 6 \%$ of cases were in the age range 62 - 81 years old $(n=3)$ and $10 \%$ of cases were in the age range $82-102$ years old $(n=5)$. Regarding sex, females accounted for $60 \%$ of cases, while males accounted for the rest of $40 \%$. Furthermore, in $90 \%$ of cases $(n=45)$, just one lower extremity was affected, whereas in $10 \%$ of cases $(n=5)$, both lower extremities were affected. Regarding thrombus location, it was found mostly in the area above the knee, particularly the popliteal vein (34\%), common femoral vein (18\%) and superficial femoral vein (20\%). Moreover, $2 \%$ of cases presented thrombus in the calf vein. In $26 \%$ of cases, multiple veins were affected. DVT was acute in $76 \%$ of cases $(n=38)$ and chronic in $24 \%$ of cases $(n=12)$. It is concluded that symptomatic and at-risk cases benefit from the use of ultrasound for DVT diagnosis. The suitability of this method stems from its lack of invasiveness and capability to assess thrombus location, magnitude and stage.
\end{abstract}

Keywords: Deep Vein Thrombosis (DVT); Post-Thrombotic Disorder (PTS); Doppler Ultrasound; Lower Limbs Ultrasound; Doppler; Deep Vein; Thrombosis; Lower limbs; Patients

\section{Overview}

Deep vein thrombosis (DVT) is a condition whereby one or multiple blood clots form in a large vein of the body, especially a vein in the muscles of the lower extremities [1]. DVT is distinguished into non-occlusive and occlusive thrombosis; the former involves partial vein obstruction, while the latter involves complete vein obstruction. The condition may only be discovered or become symptomatic when a vein is obstructed completely [2]. DVT primarily manifests as pain, swelling, redness, and vein enlargement in the affected area. However, DVT may be asymptomatic in some cases [3]. PE that could be potentially lethal and post-thrombotic disorder (PTS) are the main DVT complications $[4,5]$.

DVT is associated with high rates of morbidity and death. In the absence of appropriate treatment, DVT is particularly likely to develop (40-60\%) in cases admitted to medical orthopaedic wards with venographic DVT incidence [6]. In developing nations, it is probable that a true picture of DBT prevalence, incidence, morbid- 
ity, and mortality is lacking given that the condition can go unnoticed. The majority of affected individuals live with DVT without problems, but long-term complications can occur and venous stasis syndrome or post-phlebitic syndrome develops in almost 33\% of cases [5].

The annual number of DVT diagnoses is around 1 million. This condition is highly complex, even more so than intense myocardial dead tissue or intense stroke [7]. The manifestations of acute DVT (e.g. inflammation, pain, swelling) can be misinterpreted as infection or oedema of non-venous causes [8], leading to inconsistencies and even mistakes in clinical assessments.

Venous ultrasonography, both invasive and non-invasive, is the preferred method for diagnosing acute DVT, while the main noninvasive method of DVT diagnosis is duplex ultrasound, which is a key tool for fringe venous testing, as stipulated by the Intersocietal Commission for the Accreditation of Vascular Laboratories (ICAVL). The diagnostic efficiency of duplex ultrasound could be improved by supplementary instruments like IPG and consistent wave Doppler, but these have not been recognised so far as critical diagnostic methods as far as ICAVL accreditation is concerned [9].

The above-mentioned methods of venous ultrasonography are occasionally used interchangeably, but they vary in the sensitivity and specificity they demonstrate for acute DVT diagnosis. A range of methods are considered suitable for examination of distinctive lower furthest point veins. For instance, proximal deep veins (e.g. common femoral, femoral and popliteal veins) are usually evaluated via comparison ultrasound, while the calf and iliac veins are evaluated via a combination of duplex ultrasound and shading Doppler imaging [10].

Doppler ultrasonography was the method chosen in the present study for the examination of DVT of the lower limbs.

\section{Methodology}

Data from King Khalid Hospital in Najran spanning the period January 2019 - January 2020 were employed to conduct a retrospective descriptive quantitative analysis of the suitability of various ultrasound methods for differential diagnosis of DVT. The data derived from patients of both sexes and in the age range 20 - 102 years old who were diagnosed with different forms of DVT and who had been subjected to ultrasound assessment. The collection of the data was facilitated by a checklist based on the hospital's Picture Archive and Communication system, including patient age, sex, risk factors, and type of Doppler ultrasound. Two types of ultrasound equipment were available, namely, ultrasound Philips equipment (linear transducer 5 - 7.5 HTZ, 5 - 12 HTZ) or ultrasound Toshiba equipment (linear transducer 5 - 7.5 HTZ, 5 - 12 HTZ). Meanwhile, the ultrasound methods employed included compression ultrasound, duplex ultrasound and colour flow duplex imaging. The Statistical Package for the Social Sciences (SPSS) and Excel were the software programs used to conduct statistical analysis.

\section{Results}

As outlined in figure 1, the mean age across all cases was 46.2 \pm 19.9 years. The majority of cases $(56 \%)$ were in the age range 22 - 41 years old $(n=28)$, while $28 \%$ of cases were in the age range $42-61$ years old $(n=14), 6 \%$ of cases were in the age range 62 - 81 years old $(n=3)$ and $10 \%$ of cases were in the age range 82 102 years old $(n=5)$. Regarding sex, females accounted for $60 \%$ of cases, while males accounted for the rest of $40 \%$ (Figure 2).

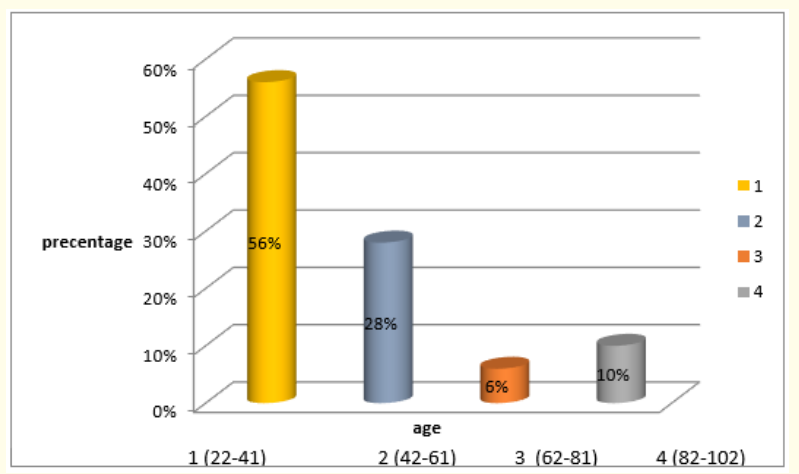

Figure 1: Frequency distribution of patients age.

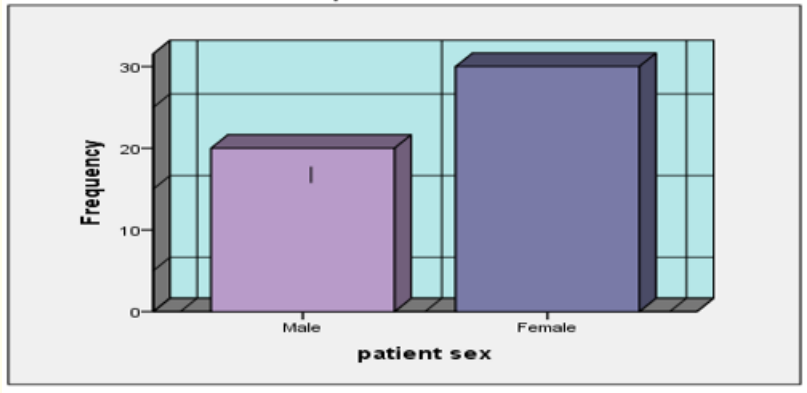

Figure 2: Frequency distribution of patient sex.

Half of the cases were subjected to compression, colour and duplex ultrasound, 14 cases were subjected to compression and du- 
plex ultrasound, and 11 cases were subjected to compression and colour Doppler (Figure 3). Furthermore, in $90 \%$ of cases $(n=45)$, just one lower extremity was affected, whereas in $10 \%$ of cases (n $=5$ ), both lower extremities were affected. Regarding thrombus location, it was found mostly in the area above the knee, particularly the popliteal vein (34\%), common femoral vein (18\%) and superficial femoral vein (20\%). Moreover, $2 \%$ of cases presented thrombus in the calf vein (Table 1 ). In $26 \%$ of cases, multiple veins were affected. DVT was acute in $76 \%$ of cases $(n=38)$ and chronic in $24 \%$ of cases ( $n=12$ ), as detailed in table 2 and 3 , respectively.

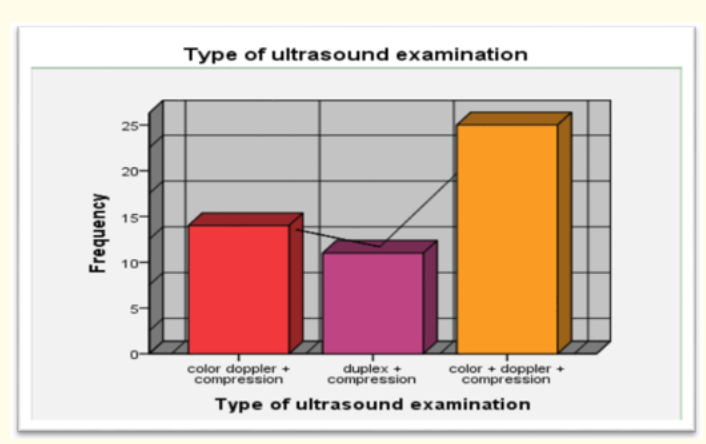

Figure 3: Frequency distribution types of ultrasound examination lower extreme.

\begin{tabular}{|l|c|c|}
\hline Site of DVT & Frequency & Percentage \\
\hline Unilateral & 45 & $90.0 \%$ \\
\hline Bilateral & 5 & $10.0 \%$ \\
\hline Total & 50 & $100.0 \%$ \\
\hline
\end{tabular}

Table 1: Frequency distribution of DVT site.

\begin{tabular}{|l|c|c|}
\hline Location of DVT & Frequency & Percentage \\
\hline Popliteal & 17 & $34.0 \%$ \\
\hline Common femoral & 9 & $18.0 \%$ \\
\hline Superficial femoral & 10 & $20.0 \%$ \\
\hline Calf & 1 & $2.0 \%$ \\
\hline More than one vein & 13 & $26.0 \%$ \\
\hline Total & 50 & $100.0 \%$ \\
\hline
\end{tabular}

Table 2: Frequency distribution of DVT location.

\begin{tabular}{|c|c|c|}
\hline DVT Type & Frequency & Percentage \\
\hline Chronic & 12 & $24.0 \%$ \\
\hline Acute & 38 & $76.0 \%$ \\
\hline Total & 50 & $100.0 \%$ \\
\hline
\end{tabular}

Table 3: Frequency distribution DVT types.

\section{Discussion}

There are significant challenges involved in diagnosing DVT due to the diversity of symptoms and manifestations, on the one hand or the lack of symptoms, on the other hand, associated with this condition. To adequately treat DVT, it is essential to have information about the characteristics of the thrombus as well as where it is located and how large it is.

In 1982, Talbot was the first to investigate the assessment of veins in the lower limbs for DVT via duplex ultrasonography. Since then, the method has become widely accepted and used as the preferred approach for DVT diagnosis. It has been confirmed to demonstrate a high level of sensitivity and specificity for assessment of symptomatic cases $[11,12]$ and positive outcomes serve as the basis for commencing anticoagulation treatment. Furthermore, duplex ultrasonography is an effective method for eliminating DVT in cases suspected of pulmonary embolus, as the latter often develops in the deep veins of the lower limbs and the initial anticoagulationbased intervention is identical.

A retrospective descriptive analysis was performed on the findings from the investigation of 50 cases of DVT assessed via B-mode and Doppler ultrasound. In each case, every major deep and superficial vein of the lower extremity, as well as the external iliac, common iliac, and IVC was investigated.

The DVT cases examined in this paper were in the age range 22 - 102 years old, with over half being in their thirties. By contrast, Hill and colleagues [13] reported that the mean age of DVT cases was 50 - 60 years old. Regarding sex, the research population in the present paper consisted mostly of females (60\%), whiles males made up the rest of $40 \%$. Meanwhile Hill and colleagues [14] reported that males accounted for the majority of DVT cases. Regarding affected extremities, the present paper found that just one lower extremity was affected in $90 \%$ of cases, whereas both lower extremities were affected in $10 \%$ of cases and there were no cases in which both lower extremities were affected despite only unilateral symptoms being displayed or in which one lower extremity was affected despite existence of bilateral symptoms. Similar results were obtained by Sheman and McArdle [15] who observed that the incidence of DVT in contra-lateral extremity was low.

Regarding thrombus location, this paper found that $34 \%$ of cases had thrombus in the popliteal vein ( $n=17), 26 \%$ of cases had thrombus in multiple veins ( $\mathrm{n}=13$ ), $20 \%$ and $18 \%$ of cases had thrombus in the superficial $(n=10)$ and common femoral vein $(n$ 
$=9$ ), respectively and $2 \%$ of cases had thrombus in the calf vein ( $\mathrm{n}$ =1). This was consistent with Markel and colleagues [16] who reported that DVT affected the proximal lower extremity more than the distal lower extremity.

In terms of DVT stage, $76 \%$ of cases in this paper were acute DVT $(n=38)$, while the rest of $24 \%$ were chronic DVT $(n=12)$. This was inconsistent with the figures reported by Hill and colleagues [12] who obtained a much lower proportion of acute DVT (17.4\%).

\section{Conclusion and Suggestion}

Various forms of DVT of the lower limb can be successfully diagnosed with different ultrasound methods. In the context of Najran Province, females are more likely than males to develop DVT. Furthermore, the popliteal vein is the affected area in the majority of cases. It is recommended that all cases suspected of DVT should be subjected to ultrasound examination. Moreover, additional prospective studies with a larger number of subjects are necessary.

\section{Bibliography}

1. Piazza G and Goldhaber SZ. "Acute pulmonary embolism: part I: epidemiology and diagnosis". Circulation 114.2 (2006): e28e32.

2. Andra H James., et al. "Tapson: 100 Questions and Answers About Deep Vein Thrombosis and Pulmonary Embolism: Copyright (C) 2008 by Jones and Bartlett Publishers, Inc (2008).

3. Kruger PC., et al. "Deep vein thrombosis: update on diagnosis and management". The Medical Journal of Australia 210.11 (2019): 516-524.

4. Blann AD and Lip GYH. "Venous thromboembolism". British Medical Journal 332 (2006): 215-219.

5. Prandoni P., et al. "The long-term clinical course of acute deep venous thrombosis". Annals of Internal Medicine 125 (1996): 1-7.

6. Geerts WH., et al. "Prevention of venous thromboembolism: American College of Chest Physicians Evidence- Based Clinical Practice Guidelines (8thedition)". Chest 133.6 (2008): 381S453S.

7. Heit JA., et al. "On behalf of the VTE Impact Assessment Group. Estimated annual number of incident and recurrent, non-fatal and fatal venous thromboembolism (VTE) events in the US". ASH Ann Meeting Aaster 106 (2005): 910.
8. Thrombosis Anthony J., et al. Sadick Published: Deep Vein Thrombosis: 2014 by Taylor and Francis Group, LLCCRC (2014).

9. Intersocietal Accreditation Commission. ICAVL: Essentials and standards for accreditation in noninvasive vascular testing". Part II. Vascular laboratory operations - Peripheral venous testing. (2000): 1-8.

10. Kearon C., et al. "Noninvasive diagnosis of deep vein thrombosis. McMaster Diagnostic Imaging Practice Guidelines Initiative". Annals of Internal Medicine 128 (1998): 663-677.

11. Killewich LA., et al. "Diagnosis of deep venous thrombosis: a prospective study comparing duplex scanning to contrast venography". Circulation 79 (1989): 810-814.

12. Comerota AJ., et al. "Venous duplex imaging: should it replace hemodynamic tests for deep venous thrombosis?" Journal of Vascular Surgery 11 (1990): 53-61.

13. Hill SL., et al. "The origin of lower extremity deep vein thrombi in acute venous thrombosis". The American Journal of Surgery 173 (1997): 485-490.

14. Hill SL., et al. "Selective use of the duplex scan in diagnosis of deep venous thrombosis". The American Journal of Surgery 170 (1995): 201-205.

15. Sheiman RG and McArdle CR. "Bilateral lower extremity US in the patient with unilateral symptoms of deep venous thrombosis: Assessment of need". Radiology 194 (1995): 171-173.

16. Markel A., et al. "Pattern and distribution of thrombi in acute venous thrombosis". Archives of Surgery 127 (1992): 305-309.

\section{Assets from publication with us}

- Prompt Acknowledgement after receiving the article

- Thorough Double blinded peer review

- Rapid Publication

- Issue of Publication Certificate

- High visibility of your Published work

Website: www.actascientific.com/

Submit Article: www.actascientific.com/submission.php Email us: editor@actascientific.com

Contact us: +919182824667 